\title{
Pragmatique et analyse des textes, [sous la direction de] Ruth Amossy
}

\section{Françoise Rigat}

\section{(2) OpenEdition}

10 Journals

\section{Édition électronique}

URL : https://journals.openedition.org/studifrancesi/41415

DOI : 10.4000/studifrancesi.41415

ISSN : 2421-5856

\section{Éditeur}

Rosenberg \& Sellier

\section{Édition imprimée}

Date de publication : 1 juillet 2004

Pagination : 237-238

ISSN : 0039-2944

\section{Référence électronique}

Françoise Rigat, «Pragmatique et analyse des textes, [sous la direction de] Ruth Amossy », Studi Francesi [En ligne], 142 (XLVIII | I) | 2004, mis en ligne le 30 novembre 2015, consulté le 09 septembre 2021. URL : http://journals.openedition.org/studifrancesi/41415; DOI : https://doi.org/10.4000/ studifrancesi. 41415

Ce document a été généré automatiquement le 9 septembre 2021.

\section{(c)}

Studi Francesi è distribuita con Licenza Creative Commons Attribuzione - Non commerciale - Non opere derivate 4.0 Internazionale. 


\title{
Pragmatique et analyse des textes, [sous la direction de] Ruth Amossy
}

\author{
Françoise Rigat
}

\section{RÉFÉRENCE}

Pragmatique et analyse des textes, [sous la direction de] RUTH AMOSSY, Presses de l'Université de Tel-Aviv, Département de français, mai 2002.

1 Ce travail réunit différentes réflexions d'une grande portée théorique et descriptive sur l'apport de la pragmatique à l'analyse textuelle des textes littéraires relevant de genres et d'époques différents tels que le théâtre, le roman, l'essai, le témoignage, la lettre (Marivaux, Proust, Robbe-Grillet). Sont regroupées ici la plupart des communications présentées lors d'un colloque organisé l'an dernier à l'université de Tel Aviv à l'initiative de Ruth Amossy et de Roselyne Koren.

2 Après une présentation exhaustive de Ruth AMOSSY, qui souligne combien l'étude des textes littéraires axée sur des phénomènes langagiers peut enrichir les sciences du langage dès lors qu'elles s'attachent à l'énoncé en situation, le volume organisé en quatre parties bien distinctes livre en première partie des mises au point fondamentales sur la question du dispositif d'énonciation, et notamment dans le récit.

Gilles PHILIPPE («L'appareil formel de l'effacement énonciatif et la pragmatique des textes sans locuteur») définit, plus qu'il ne décrit dans le détail, la notion d'un «appareil formel de la non-énonciation». Certains textes comme l'annuaire, le code civil et d'autres textes narratifs plus littéraires (la prose de Flaubert ou de Sartre par exemple) qui effacent toute trace de la subjectivité présentent un contrat de lecture spécifique où la légitimité et l'interprétation dépendent justement de l'absence de locuteur et d'ethos textuel. L'auteur conclut à la nécessité d'une étude approfondie de cette notion, à savoir «l'appareil formel de la non-énonciation», tout en reprochant à la pragmatique, voire à la stylistique, de s'être par trop arrêtée sur l'évaluation des traces énonciatives, au détriment de l'analyse des faits d'effacement du sujet dans le discours. 
4 L'article de Ruth Amossy précise en quoi deux disciplines apparemment fort diverses la narratologie et la pragmatique - ont partie liée («De l'énonciation à l'interaction: l'analyse du récit entre pragmatique et narratologie»). A partir d'exemples puisés dans l'œuvre de Robert Pinget, elle montre comment la pragmatique prise sous l'angle interactionnel - dialogique - permet l'analyse du récit littéraire en termes d'échange : non pas le texte narratif objet de la poétique, d'une part, et le lecteur, objet d'une théorie de la réception de l'autre, mais une relation dynamique lecteur-texte. L'étude du récit de fiction dans une telle perspective ne peut que dynamiser les propositions de la narratologie classique.

5 Le deuxième volet d'études porte sur l'hétérogénéité discursive, c'est-à-dire «la capacité de mettre en scène plusieurs voix simultanément». Plusieurs exemples tirés du récit, du théâtre ainsi que de l'essai-manifeste permettent de couvrir le champ linguistique (les effets de la polyphonie) mais aussi esthétique (la question du style).

6 L'article de Anna JAUBERT («Enonciation clivée et discours littéraire: la pragmatique à large spectre des vrais et faux reports de voix») interroge la reconnaissance du discours rapporté lorsqu'il n'est pas signalé comme tel: le discours indirect libre bien sûr, mais aussi le discours direct libre, moins connu (un discours direct qui n'est marqué ni typographiquement, ni par un verbe) et qui cependant recouvre un traitement original de l'hétérogénéité énonciative. L'auteure montre d'abord que l'effet de bivocalité (ou énonciation double) du discours indirect libre dans le récit n'est pas uniquement corrélé à certains indices forts (comme l'imparfait), mais peut être assuré par la cohérence discursive. Pour le discours, par contre, c'est le théatre (Marivaux), haut lieu de l'interaction conversationnelle, qui lui offre de nombreux exemples de ce qu'elle nomme les «degrés intermédiaires d'appropriation de la parole de l'autre». Quant au discours direct libre analysé dans le monologue intérieur, elle souligne qu'il s'agit plus d'une «pensée rapportée» que d'un discours rapporté, difficile parfois à distinguer de l'ironie.

7 Isabelle SERCA («Ponctuation et énonciation: guillemets, parenthèses et discours rapporté chez Proust») analyse par le biais de la ponctuation les diverses modalités mises en œuvre par Marcel Proust pour donner la parole à ses personnages. Ainsi utilise-t-il les guillemets ou l'italique pour restituer les propos de ses personnages avant d'en proposer entre parenthèses une analyse métalinguistique qu'attestent les expressions comme «mon interlocuteur voulait dire», «en donnant au mot [...] le sens de» etc. Il suffit de penser à l'idiolecte de Françoise dont le Narrateur s'est fait le miroir, ou encore aux nombreuses incises, apposées entre tirets : tous ces signes renforcent la dimension polyphonique, créant ce «feuilleté» typiquement proustien. Loin d'être de simples artifices typographiques, ces signes sont non seulement l'un des éléments essentiels de la stratégie énonciative de l'auteur de la Recherche, mais permettent également d'en apprécier la stratégie littéraire, d'en appréhender le «style».

Dans «L'écriture polyphonique de l'essai littéraire: l'exemple de Robbe-Grillet», Galia YANOSCHEVSKY ne se limite pas à l'analyse du discours rapporté, mais prend en compte l'intertexte ainsi que les différentes versions d'un discours par essence monologal: l'essai. Son travail porte sur les différentes stratégies discursives adoptées par RobbeGrillet pour mettre en scène les voix protagonistes et antagonistes dans la polémique qui s'est déroulée autour du Nouveau roman. Le potentiel de l'hétérogénéité discursive (et sa dimension argumentative) est ici clairement mis en relief: l'auteure analyse entre autres comment Robbe-Grillet fonde son discours en faisant appel à l'autorité (qu'elle 
soit explicite - Sarraute - ou implicite - Barthes -) ou en rapportant les paroles de ses détracteurs pour renforcer sa thèse.

9 La troisième section du volume s'attache à montrer comment les recherches sur les actes de discours peuvent enrichir l'analyse des textes.

10 Gisèle VALENCY SLAKTA reprend les outils conceptuels proposés par Searle pour analyser le jeu de mots et le malentendu dans le récit balzacien ("Actes de discours indirects et double adresse: malentendus et jeux de mots»). Le fonctionnement langagier de ces phénomènes est expliqué à la lumière de la notion de «double adresse»: celle-ci indique que le locuteur est comme un tiers dans un dialogue où les deux interlocuteurs font de la position discursive de l'un d'entre eux un objet distinct (lorsque, par exemple, un professeur dit à ses élèves «on ne copie pas»). Cette spécificité des jeux de mots aboutit à une réflexion plus vaste, puisque leur interprétation pose un problème aux théories de la narration: il s'agit de savoir si le tiers défini par la double adresse est assimilable à la troisième personne fictionnelle.

11 Carole DoRNIER, de son côté, envisage le récit de témoignage comme un acte de parole dans la mesure où il engage son locuteur sur la vérité de ce qu'il rapporte («Verbes assertifs à la première personne et pragmatique du témoignage dans les Mémoires de Valentin Jamerey-Duval»). Ainsi s'appuient-elle sur les concepts de performatif de Austin et d'acte de langage de Searle pour relever les marques linguistiques qui construisent la crédibilité du discours testimonial. Les mémoires d'un auteur méconnu du XVIIIe siècle fournissent de nombreux exemples de verbes assertifs qui expriment l'attitude du locuteur à l'égard des informations: c'est le cas des verbes tels que «j'ai appris que», «j'ai su que» qui "présupposent la vérité du contenu de la complétive qui suit et font admettre au lecteur la vision du monde qui est celle du narrateur».

La contribution de Roselyne KOREN relance la réflexion sur l'apport de la pragmatique dans l'étude de la spécificité de l'écriture littéraire, du «narrateur neutre» notamment («Parole littéraire, légitimation et désengagement: de quelques paradoxes tout à fait admissibles»). Par le biais de concepts pragmatiques de commitment (Searle) et de responsabilité de l'énonciateur, elle s'intéresse à la valeur de vérité du texte littéraire, et s'interroge sur la neutralité qu'affichent certaines textes portant sur des dilemmes éthiques dans plusieurs extraits de romans consacrés au terrorisme et parus dans le Monde diplomatique.

13 La quatrième et dernière partie de l'ouvrage présente deux travaux qui montrent comment les outils pragmatiques éclairent le fonctionnement d'un type de discours jusque-là étudié par la rhétorique : l'épistolaire, ici la correspondance diplomatique et la lettre amoureuse au siècle des Lumières.

Pour dégager les différents niveaux de l'interaction, Sivane COHEN («Etude taxémique d'une correspondance diplomatique: images de la France et de l'Allemagne après la guerre de 1870») utilise une notion de linguistique conversationnelle, les taxèmes - qui marquent la position haute ou basse dans l'interaction - ; par exemple l'initiative (position haute) apparaît de façon nette «j'ai exprimé», «je me récriai» etc. L'auteure met en évidence comment un même entretien est modulé selon les rapports de place entre les correspondants (le chancelier Bismarck et l'ambassadeur de France) et leurs objectifs argumentatifs. Ici la pragmatique replace le genre épistolaire dans une perspective interactionnelle et non monologale. 
Quant à Jürgen SIESS («Eléments pour une approche pragmatique du discours épistolaire au XVIIIe siècle»), il explore les éléments interactionnels, l'ensemble de normes d'écriture et de règles de comportement qui dans la correspondance de l'Ancien Régime, sont en grande partie codifié dans les manuels. Il dégage ainsi «une dynamique portée par les projets respectifs des épistoliers tels qu'ils peuvent se former et se dire» à travers les contraintes issues de leur sexe, de leur âge, de leur compétence culturelle et de leur rang.

En conclusion, on redira tout l'intérêt de cet ouvrage, que l'on ne saurait trop recommander aux linguistes-littéraires pour les pistes de recherche tout à fait stimulantes qu'il indique. Sous des angles divers (acte de langage, ponctuation, narratologie etc.) ces études montrent comment la linguistique du discours peut nourrir la recherche littéraire et, vice versa, comment le texte littéraire permet de cerner des fonctionnements langagiers que «l'usage du discours ordinaire laisse généralement dans l'ombre». Un regret tout de même: sans doute le livre aurait-il gagner à préciser dès le titre qu'il s'agissait de textes essentiellement littéraires. 